Results The use of the EKOS catheter and TPA infusion restored sufficient venous drainage and ultimately the patient developed good collateral flow. The result was an excellent six-month post-operative neurologic outcome.

Conclusion CVST can be refractory to existing endovascular treatment options. We describe the use of an EKOS catheter, traditionally used in peripheral vascular and pulmonary emboli cases, as rescue therapy in a cerebrovascular case where traditional treatment options failed. Further evaluation is warranted to clarify its safety and efficacy versus other therapeutic modalities.

Disclosures N. Vivelo: None. R. Ryan: None. R. SanchezMejia: None.

\section{E-064 SURPASS EVOLVE FLOW DIVERSION FOR EMBOLIZATION OF UNRUPTURED INTRACEREBRAL ANEURYSMS: EARLY EXPERIENCE OF A SINGLE TERTIARY CENTER}

L Verhey*, A Restrepo Orozco, L Lyons, A Sewell, J Tsai, P Mazaris, J Singer. Neurological Surgery, Spectrum Health, Michigan State University, Grand Rapids, MI

10.1136/neurintsurg-2021-SNIS.159

Introduction Since initially developed, flow-diverting devices for intracerebral endoluminal reconstruction have rapidly evolved and multiple devices now exist, differing in materials and design. Recently, the Stryker Surpass Evolve flow-diverter (FD) has been approved as a 64-wire cobalt-chromium device with improved ease of deployment, flexibility and conformability compared to its predecessor.

Objective To summarize the clinical applications and procedural outcomes of patients who underwent Surpass Evolve FD embolization of an unruptured intracerebral aneurysm at a tertiary high-volume single center

Methods The first Surpass Evolve FD was deployed at our institution on August 31, 2020. All consecutive patients who presented since this date with a symptomatic or asymptomatic unruptured intracerebral aneurysm and were treated with a Surpass Evolve FD were included. Patients with a ruptured intracerebral aneurysm were excluded. Demographic and clinical features, antithrombotic therapy, aneurysm features, access, procedural factors and outcome, and post-procedural neurologic status were extracted from our departmental neurointerventional database and electronic medical records. Follow-up cerebral angiograms were also reviewed. Summary statistics included frequencies, and measures of central tendency and dispersion as appropriate.

Results Since Aug 31, 2020, 32 patients underwent embolization of an unruptured intracerebral aneurysm with the Surpass Evolve, and have been followed for a mean of 118.9 (SD 62.3) days. Mean age at intervention was 62.5 (13.4) years, and $25(78 \%)$ were female. In $27(84 \%)$ patients, a native aneurysm was flow-diverted, and in 5 (16\%) patients, FD was performed for a recurrent aneurysm. Distribution of aneurysm location was: ICA 21 (66\%), ACA 2 (6\%), AComm 2 (6\%), and posterior fossa 7 (22\%). Mean aneurysm size was 8.8 (4.7) $\mathrm{mm}$. Access was radial in $23(72 \%)$, femoral in $8(25 \%)$ and ulnar in $1(3 \%)$. FD was successfully deployed to the target in $31(97 \%)$ of cases. Median FD per patient was one; nine patients had two FD devices placed. Balloon angioplasty or J-wire manipulation was used to enhance apposition in 19 $(59 \%)$ cases. Neurologic deficits were observed in four patients; 3 (9\%) had transient deficits, and 1 (3\%) had a permanent deficit. An asymptomatic microperforation was observed in one patient. Migration of the FD was observed in one patient following balloon angioplasty. Early contrast stasis was observed in all patients, and short-term follow-up angiogram for three patients indicated decreased aneurysm size.

Conclusion In our early experience, the Surpass Evolve FD is safe and has an improved ease-of-use profile compared with its Surpass Streamline predecessor. Complication rates are well within the acceptable limits based on published evidence. Serial follow-up angiography will delineate the rate of aneurysm obliteration.

Disclosures L. Verhey: 1; C; Stryker Neurovascular. A. Restrepo Orozco: None. L. Lyons: None. A. Sewell: None. J. Tsai: None. P. Mazaris: None. J. Singer: 2; C; Stryker, Medtronic, Penumbra, Nico, Cerenovus.

\section{E-065 STENTING AND FLOW DIVERSION FOR PEDIATRIC INTRACRANIAL ANEURYSMS}

H Rai ${ }^{*}$, F Almayman, M Waqas, A Kalanovic, A Monteiro, A Baig, M Recker, A Siddiqui, E Levy. Neurosurgery, Uni at Buffalo, Buffalo, NY

\subsection{6/neurintsurg-2021-SNIS. 160}

Background Pediatric intracranial aneurysms (IA) are rare. Literature highlights endovascular treatment yielding favorable outcomes, but long-term follow up outcomes are lacking.

Objective To analyze our institution's experience of endovascular management of pediatric cerebral aneurysms with longterm follow-up.

Methods In the past decade, 4 male and 2 female patients with nine IA were treated with stenting or flow-diversion. We retrospectively reviewed the radiological, hospital and outpatient clinic charts to document clinical presentation, diagnostic methods, treatment strategies, outcome and long-term follow up for each patient.

Results Headache was the presenting symptom in all of the patients except one, which had a traumatic injury. Two of these patients had a ruptured aneurysm. Two patients had multiple aneurysms - 2 and 3 respectively. The aneurysms were most frequently located in the anterior circulation, with the exception of two basilar. Four of the aneurysms were treated with stenting, while 5 with Pipeline flow-diversion. All cases, except one, had a good outcome after the procedure; the latter having a hemorrhage, requiring hematoma evacuation, and later developing in-stent stenosis, for which balloon angioplasty was done and another pipeline flow-diverter was deployed. Long term imaging follow-up demonstrated no issues regarding the durability of flow-diverters and stents, with 0 modified Rankin Score (mRS) reported for 5 patients and a baseline of 2 for one patient.

Conclusion This series demonstrates the safety and effectiveness of endovascular treatment of pediatric aneurysms, with no issues regarding the durability of flow-diverters and stents at continued long-term follow up.

Disclosures H. Rai: None. F. Almayman: None. M. Waqas: None. A. Kalanovic: None. A. Monteiro: None. A. Baig: None. M. Recker: None. A. Siddiqui: 1; C; NIH/NINDS 1R01NS091075. 4; C; Amnis Therapeutics, Apama Medical, Blink TBI, Buffalo Technology Partners, Cardinal Consultants, Cerebrotech Medical Systems, Cognition Medical, Endostream Medical, Imperative Care, International Medi. E. Levy: 2; C; 